\title{
The establishment and application of preimplantation genetic haplotyping in embryo diagnosis for reciprocal and Robertsonian translocation carriers
}

Shuo Zhang ${ }^{1,3}$, Caixia Lei ${ }^{1,2}$, Junping Wu ${ }^{1,2}$, Jing Zhou ${ }^{1}$, Haiyan Sun ${ }^{1}$, Jing Fu ${ }^{1,2}$, Yijuan Sun ${ }^{1}$, Xiaoxi Sun ${ }^{1,2^{*}}$, Daru Lu ${ }^{3^{*}}$ and Yueping Zhang ${ }^{1,2^{*}}$

\begin{abstract}
Background: Preimplantation genetic diagnosis (PGD) is now widely used to select embryos free of chromosomal copy number variations (CNV) from chromosome balanced translocation carriers. However, it remains a difficulty to distinguish in embryos between balanced and structurally normal chromosomes efficiently.

Methods: For this purpose, genome wide preimplantation genetic haplotyping (PGH) analysis was utilized based on single nucleotide polymorphism (SNP) microarray. SNPs that are heterozygous in the carrier and, homozygous in the carrier's partner and carrier's family member are defined as informative SNPs. The haplotypes including the breakpoint regions, the whole chromosomes involved in the translocation and the corresponding homologous chromosomes are established with these informative SNPs in the couple, reference and embryos. In order to perform this analysis, a reference either a translocation carrier's family member or one unbalanced embryo is required. The positions of translocation breakpoints are identified by molecular karyotypes of unbalanced embryos. The recombination of breakpoint regions in embryos could be identified.
\end{abstract}

Results: Eleven translocation families were enrolled. 68 blastocysts were analyzed, in which 42 were unbalanced or aneuploid and the other 26 were balanced or normal chromosomes. Thirteen embryos were transferred back to patients. Prenatal cytogenetic analysis of amniotic fluid cells was performed. The results predicted by PGH and karyotypes were totally consistent.

Conclusions: With the successful clinical application, we demonstrate that PGH was a simple, efficient, and popularized method to distinguish between balanced and structurally normal chromosome embryos.

Keywords: Single nucleotide polymorphism, Breakpoint, Preimplantation genetic haplotyping, Reciprocal translocation, Robertsonian translocation

\section{Background}

Balanced translocation is a relatively common structural chromosome rearrangement that occurs when an exchange of terminal segments happens between different chromosomes, including Reciprocal and Robertsonian

\footnotetext{
* Correspondence: steven3019@hotmail.com; drlu@fudan.edu.cn; jiaitg@163.com

${ }^{1}$ Shanghai Ji Ai Genetics \& IVF Institute, Obstetrics and Gynecology Hospital, Fudan University, 588 Fangxie Rd, Shanghai 200011, China

${ }^{3}$ Collaborative Innovation Center for Genetics and Development, School of Life Sciences, Fudan University, 2005 Songhu Rd, Shanghai 200438, China

Full list of author information is available at the end of the article
}

translocation [1]. It has an estimated prevalence of $0.19 \%$ in the general population and $2.2 \%$ in patients who undergo in vitro fertilization (IVF) treatment and experience a history of recurrent miscarriages or repeated IVF failure [2]. Although carriers of balanced chromosomal rearrangements usually have a normal phenotype, the risk of producing unbalanced gametes is high due to complex segregation modes during meiosis [3]. A quadrivalent structure is formed at meiosis I through pairing of translocated chromosomes and the two corresponding normal chromosomes. This structure 
commonly undergoes one of the three modes of segregation: $2: 2,3: 1$ or $4: 0$. Only the alternate segregation pattern in 2:2 mode can result in normal or balanced gametes, the other segregation patterns will generate unbalanced types of gametes which can lead to apparent infertility, recurrent miscarriage, or delivery of a phenotypically abnormal offspring with mental retardation or other congenital abnormalities $[4,5]$.

For couples with a known balanced chromosome translocation, preimplantation genetic diagnosis (PGD) with fluorescent in-situ hybridization (FISH) has been used to select normal/ balanced embryos for transfer [6, 7]. However, FISH is limited by technical difficulties such as cell fixation and signal interpretation [8]. In recent years, microarray technologies and next-generation sequencing (NGS) which are capable of testing for chromosome translocation imbalances and screening for aneuploidy of all 23-pairs of chromosomes simultaneously have been widely used in many centers [9-12]. Studies performed previously indicate that array-based PGD can improve pregnancy rates in translocation carrier couples to between $45 \%-70 \%$ per transfer [12-14]. Despite this advantage, these traditional PGD methods can't distinguish balanced and structurally normal embryos from translocation carriers. To date, some feasible technologies have been introduced to solve this difficulty. FISH with specific probes spanning the chromosome breakpoints was used earlier $[15,16]$. Treff and colleagues enrolled reciprocal translocation families and predicted the balanced or structurally normal embryos based upon genotype within five $\mathrm{Mb}$ of the breakpoints [17]. The unbalanced embryos were used as a reference. In addition, in the latest research of $\mathrm{Hu}$ et al. [18], the authors developed a "MicroSeq-PGD" method which combined chromosome microdissection and NGS in reciprocal translocation carriers to characterize the DNA sequence of the translocation breakpoints to distinguish embryos. However, these methods are possibly time-consuming, complicated, and homologous recombination can't be identified. More importantly, Robertsonian translocation carriers are not included, which have an estimated $0.1 \%$ incidence rate in the general population [19].

Therefore, the most suitable methodological design for translocation carriers has not been well established. Preimplantation genetic haplotyping (PGH) was first introduced for preimplantation genetic testing of single gene defects by polymorphic short tandem repeat (STR) markers [20]. In this current study, our aim was to utilize PGH to distinguish between balanced and structurally normal embryos prior to implantation from both reciprocal translocation and Robertsonian translocation carriers accurately, along with the genetic screening for all 23-pairs of chromosomes. For this purpose, a SNP microarray was used [21]. Haplotypes including the breakpoint regions, the whole chromosomes involved in the translocation and corresponding normal homologous chromosomes were established using informative SNP markers. The carrier's family member or an unbalanced embryo was used as a reference. The laboratory technicians were blinded regarding the PGH results. The predictive accuracy of PGH was validated by the conventional amniotic fluid karyotypes in the second trimester.

\section{Methods \\ Patients}

Eleven translocation carrier families that would undergo assisted reproductive were enrolled in Shanghai Ji Ai Genetics \& IVF Institute from June 2014 to Marth 2016. All families had a history of a recurrent spontaneous abortion, infertility or pregnancies with chromosome anomalies. The translocation karyotypes were 46,XY, $\mathrm{t}(5 ; 22) \quad(\mathrm{q} 33 ; \quad \mathrm{q} 12) ; \quad 46, \mathrm{XX}, \mathrm{t} \quad(16 ; \quad 18) \quad(\mathrm{q} 22 ; \mathrm{q} 21.1)$; 46,XX,t(12;22)(p12;q13); 46,XX,t(11;16) (p11.2; p13.1); 46, XY, t (1;19) (q12; p13); 45,XX, rob(14;21) (q10;q10); $45, \mathrm{XY}, \operatorname{rob}(14 ; 21)$ (q10; q10); 45, XX, rob $(14 ; 15)$ (q10;q10); 46,XY,t(6;9)(q27;q22); 46,XX,t(2;3) (q22.1; p14.1); 46,XY, t(7; 11) (q21;q21), respectively. Ten $\mathrm{ml}$ peripheral blood from each couple and family members was collected at recruitment. Written informed consent was obtained from each family and the study protocol was approved by the Ethics Committee for Human Subject research of the Obstetrics and Gynecology Hospital, Fudan University.

\section{Blastocyst biopsy and WGA}

For embryos at the blastocyst stage, three to ten cells were removed from the trophectoderm on day five or six of embryonic development. The biopsied cells were placed into polymerase chain reaction tubes with an alkaline denaturation buffer for cell lysis as previously describe. Whole genomic amplification (WGA) was performed by the multiple displacement amplification (MDA) method. Isothermal DNA amplification with phi 29 DNA polymerase was performed (Repli-g single cell kit, QIAGEN GmbH, Hilden, Germany) as described in the manufacturers' protocol. The isothermal amplification was performed at $30{ }^{\circ} \mathrm{C}$ for $8 \mathrm{~h}$ and the reaction was stopped by incubation at $65{ }^{\circ} \mathrm{C}$ for $3 \mathrm{~min}$.

\section{SNP-array and analysis}

SNP genotypes were performed with Illumina Human Karyomap-12 V1.0 microarray in this study as previously described [21]. Each Karyomap-12 bead chip contained approximately 300,000 SNPs. Molecular karyotypes and haplotypes could be established with this method simultaneously in each embryo. This information was then used to identify the normal embryos free of chromosomal copy number variations $(\mathrm{CNV})$ and distinguish 
between balanced and structurally normal embryos respectively. The molecular karyotype analysis and the linkage analysis of haplotype were performed with Bluefuse-Multi software (Illumina, Inc. San Diego, USA).

Informative SNPs were used to establish the haplotypes including the breakpoint regions, the whole chromosomes involved in the translocation and the corresponding normal homologous chromosomes in the couple, reference and embryos. The informative SNPs of $\pm 2 \mathrm{Mb}$ around the breakpoints were selected to establish haplotypes of the regions covering the breakpoints. The selection criteria for informative SNPs, was that they should be heterozygous in the carrier and homozygous in his/her partner. Also these SNPs should be homozygous in the carrier's parents or other family members. This information was used to determine which of the carrier's two haplotypes were linked to the derivative chromosome or to the normal chromosome. The carrier's family member or an unbalanced embryo was used as a reference. If the carrier's unbalanced embryo was used as a reference, then there was no requirement to study family members.

The haplotypes of the whole chromosomes involved in the balanced translocation and the corresponding normal homologous chromosomes could indicate the presence of homologous recombination around the breakpoints. The predictive criterion to distinguish balanced and structurally normal embryos combined the haplotypes of breakpoint regions and the presence of homologous recombination in these regions. When recombination doesn't occur, if the embryo carries the same haplotype with the carrier's family member who has the same translocation or unbalanced embryos, or if the embryo carries the different haplotype with the carrier's family member who has the normal karyotype, and therefore it will be defined as a balanced translocation embryo; If the embryo carries the same haplotype with the carrier's family member who has the normal karyotype, or if the embryo carries the different haplotype with the carrier's family member who has the same translocation or unbalanced embryos, then it will be defined as a structurally normal embryo. When recombination occurs, the result might be hard to predict due to the complexity. The consistency of predictive results with different reference samples including family members and unbalanced embryos was compared. The predictive accuracy of $\mathrm{PGH}$ was validated by a blinded comparison with conventional amniotic fluid cell karyotypes in the second trimester of successful pregnancies after embryo transfer.

\section{Results}

In this study, the 11 balanced translocation families underwent 14 IVF cycles. Family10 underwent three cycles, and the other families had one cycle each. The characteristics and ovarian stimulation results of these patients are listed in Table 1. In families 1-8, the translocation was inherited from a parent. In family 9, the carrier's parents had already died and the couple didn't have unbalanced embryos, but the carrier's sister and brother were both identified to carry the same translocation. In family 10 and 11 , the two couples didn't tell

Table 1 The characteristics of the patients in this study

\begin{tabular}{|c|c|c|c|c|c|c|c|c|}
\hline Family & $\begin{array}{l}\text { Maternal age } \\
\text { /Paternal age }\end{array}$ & Karyotype $^{a}$ & $\begin{array}{l}\text { Reason for } \\
\text { karyotyping }\end{array}$ & $\begin{array}{l}\text { The number of } \\
\text { oocytes retrievedf }\end{array}$ & $\begin{array}{l}\text { The number of } \\
\text { mature oocytes (MII) }\end{array}$ & $\begin{array}{l}\text { The number of } \\
\text { fertilized oocytes }\end{array}$ & $\begin{array}{l}\text { The number of } \\
\text { D3 oocytes }\end{array}$ & $\begin{array}{l}\text { The number of } \\
\text { biopsied blastocysts }\end{array}$ \\
\hline 1 & $33 / 34$ & $46, X Y, t(5 ; 22)(q 33 ; q 12) \mathrm{mat}$ & Infertility & 16 & 15 & 15 & 15 & 5 \\
\hline 2 & $33 / 30$ & $46, X X, t(16 ; 18)(q 22 ; q 21.1)$ pat & $\begin{array}{l}\text { repeated } \\
\text { miscarriage }\end{array}$ & 21 & 16 & 15 & 11 & 4 \\
\hline 3 & $23 / 31$ & $46, X X, t(12 ; 22)(p 12 ; q 13)$ pat & $\begin{array}{l}\text { repeated } \\
\text { miscarriage }\end{array}$ & 14 & 8 & 5 & 5 & 3 \\
\hline 4 & $29 / 31$ & $46, X X, t(11 ; 16)(p 11.2 ; p 12.3)$ pat & $\begin{array}{l}\text { affected } \\
\text { fetus }\end{array}$ & 15 & 13 & 9 & 9 & 6 \\
\hline 5 & $29 / 29$ & $46, X Y, t(1 ; 19)(q 12 ; p 13)$ mat & $\begin{array}{l}\text { repeated } \\
\text { miscarriage }\end{array}$ & 19 & 14 & 12 & 11 & 6 \\
\hline 6 & $27 / 26$ & $45, X X, \operatorname{rob}(14 ; 21)(q 10 ; q 10)$ mat & $\begin{array}{l}\text { repeated } \\
\text { miscarriage }\end{array}$ & 18 & 12 & 7 & 7 & 6 \\
\hline 7 & $31 / 33$ & $45, X Y, \operatorname{rob}(14 ; 21)(q 10 ; q 10)$ pat & Infertility & 16 & 16 & 16 & 13 & 8 \\
\hline 8 & $26 / 29$ & $45, X X, \operatorname{rob}(14 ; 15)(q 10 ; q 10)$ pat & $\begin{array}{l}\text { repeated } \\
\text { miscarriage }\end{array}$ & 4 & 3 & 3 & 3 & 3 \\
\hline 9 & $36 / 53$ & $46, X Y, t(6 ; 9)(q 27 ; q 22) \mathrm{mat} / \mathrm{pat}$ & $\begin{array}{l}\text { repeated } \\
\text { miscarriage }\end{array}$ & 8 & 8 & 5 & 5 & 3 \\
\hline $10^{\mathrm{b}}$ & $29 / 30$ & $46, X X, t(2 ; 3)(q 22.1 ; p 14.1)$ & $\begin{array}{l}\text { repeated } \\
\text { miscarriage }\end{array}$ & 31 & 26 & 26 & 21 & 12 \\
\hline 11 & $25 / 28$ & $46, X Y, t(7 ; 11)(q 21 ; q 21)$ & Infertility & 28 & 28 & 26 & 22 & 12 \\
\hline
\end{tabular}

${ }^{a}$ The karyotypes were identified by peripheral blood cells

In family-10, the number of oocytes was from 3 cycles 
their parents that they were undergoing IVF treatment and the carrier was an only child, therefore we couldn't get the peripheral blood karyotype of their family members. While they expressed a strong desire to transfer the structurally normal embryo, therefore the unbalanced embryos were used as reference. With our method, we obtained molecular karyotypes from all the 68 biopsied blastocysts. Of the 68 diagnosed blastocysts, 26 were balanced or normal, 25 blastocysts had translocation related abnormalities and 17 blastocysts showed de novo abnormalities unrelated to the translocation. PGH analysis then was performed in the 26 blastocysts that were balanced or normal, which indicated that 12 were balanced and 14 were structurally normal embryos. The predictive results of two different breakpoint regions in each embryo were consistent with the amniotic fluid analysis in ongoing pregnancies.

Haplotypes were assigned for the carrier, the partner, the embryos and the carrier's family member. The molecular karyotypes of unbalanced embryos could help to pinpoint the relatively accurate position of breakpoint. If the breakpoint couldn't be identified by unbalanced embryos, then that from the peripheral blood karyotype was used. Actually, the haplotype of any region or chromosome genome wide could be established and therefore this method is universal for any kind of translocation. Detailed results of the microarray platform of the transferred blastocysts were shown in
Table 2, the other non- transferred embryos are in Additional file 1: Table S1.The process of establishing haplotypes and distinguishing between balanced and structurally normal chromosome embryos through PGH analysis was shown in Fig. 1. The haplotypes of two breakpoint regions and the chromosomes involved in the translocation and the normal homologous chromosomes in family 3 are shown in Fig. 2. The summary of informative SNPs that were used to establish the whole haplotypes of the successfully transferred blastocysts is listed in Table 3, the other non- transferred embryos are in Additional file 2: Table S2. In family 2, 5,6 and 7, the predictive results using family members or unbalanced embryos with deletion were consistent, which was shown in Table 2. The other families had either no unbalanced embryos with deletion or no available family members, then the comparison of predictive results couldn't be performed.

When finishing the PGH analysis, part of the families had already had their embryos transferred. Fourteen blastocysts were thawed and the transfer cycles were performed. In family 10, the women failed to achieve pregnancy in the first and second transfer cycle, while she was successful in the third cycle. In the other families, all became pregnant in the first cycle. For all the women that were pregnant after embryo transfer, cytogenetic analysis of amniotic fluid was required to be performed in the second trimesters (Table 2). We proved

Table 2 Detailed results of microarray platform of the transferred blastocysts

\begin{tabular}{|c|c|c|c|c|c|c|c|c|}
\hline \multirow[t]{2}{*}{ Family } & \multirow{2}{*}{$\begin{array}{l}\text { The number of } \\
\text { biopsied blastocysts }\end{array}$} & \multirow{2}{*}{$\begin{array}{l}\text { Grade of } \\
\text { blastocysts }\end{array}$} & \multirow{2}{*}{$\begin{array}{l}\text { Molecular } \\
\text { karyotype }\end{array}$} & \multicolumn{2}{|l|}{ Results of PGH } & \multirow{2}{*}{$\begin{array}{l}\text { Transferred } \\
\text { blastocysts }\end{array}$} & \multirow{2}{*}{$\begin{array}{l}\text { Karyotype of } \\
\text { amniotic fluid }\end{array}$} & \multirow[t]{2}{*}{ Consistency? } \\
\hline & & & & Family member $^{a}$ & Unbalanced embryos $^{\mathrm{b}}$ & & & \\
\hline 1 & 7 & $6 \mathrm{BB}$ & $(1-22, X) * 2$ & Carrier & NA & Embryo-1 & $46, X X, t(5 ; 22)(q 33 ; q 12)$ & Yes \\
\hline 2 & 4 & $5 \mathrm{BC}$ & $(1-22, X) * 2$ & Normal & Normal & Embryo-4 & $46, X X$ & Yes \\
\hline 3 & 3 & $5 B B$ & $\begin{array}{l}(1-22)^{*} 2 \\
(X Y)^{* 1}\end{array}$ & Carrier & NA & Embryo-6 & $46, X Y, t(12 ; 22)(p 12 ; q 13)$ & Yes \\
\hline 4 & 6 & $5 B B$ & $(1-22, X)^{*} 2$ & Normal & NA & Embryo-1 & $46, X X$ & Yes \\
\hline 5 & 6 & $5 A B$ & $(1-22, X) * 2$ & Carrier & Carrier & Embryo-1 & $46, X X, t(1 ; 19)(q 12 ; p 13)$ & Yes \\
\hline 6 & 6 & $5 \mathrm{BC}$ & $(1-22, X) * 2$ & Carrier & Carrier & Embryo-9 & $45, X X, \operatorname{rob}(14 ; 21)(q 10 ; q 10)$ & Yes \\
\hline 7 & 8 & $5 \mathrm{BC}$ & $(1-22, X) * 2$ & Normal & Normal & Embryo-6 & $46, X X$ & Yes \\
\hline 8 & 3 & $5 B B$ & $\begin{array}{l}(1-22)^{*} 2 \\
(X Y)^{* 1}\end{array}$ & Carrier & NA & Embryo-2 & $45, X Y, \operatorname{der}(14 ; 15)(q 10 ; q 10)$ & Yes \\
\hline 9 & 3 & $5 A B$ & $(1-22, X) * 2$ & Normal & NA & Embryo-4 & $46, X X$ & Yes \\
\hline \multirow[t]{3}{*}{10} & 12 & $5 \mathrm{AC}$ & $(1-22, X) * 2$ & NA & Carrier & Embryo-1-5 & No pregnancy & NA \\
\hline & & $5 B B$ & $(1-22, X) * 2$ & NA & Carrier & Embryo-3-4 & No pregnancy & NA \\
\hline & & $5 \mathrm{BC}$ & $\begin{array}{l}(1-22)^{*} 2 \\
(X Y)^{* 1}\end{array}$ & NA & Normal & Embryo-3-1 & $46, X Y$ & Yes \\
\hline 11 & 12 & $5 \mathrm{BC}$ & $(1-22, X) * 2$ & NA & Normal & Embryo-12 & $46, X X$ & Yes \\
\hline
\end{tabular}

NA = not available

an family $1-9$, the family member was used as a reference; in family10-11, the unbalanced embryo was used as a reference

bIn family2, embryo-1, embryo-4 and embryo-9 were included; In family5, embryo-3 was included; In family6, embryo-4 was included; In family7, embryo-13 was included; In other families, the unbalanced embryos couldn't be used as reference 


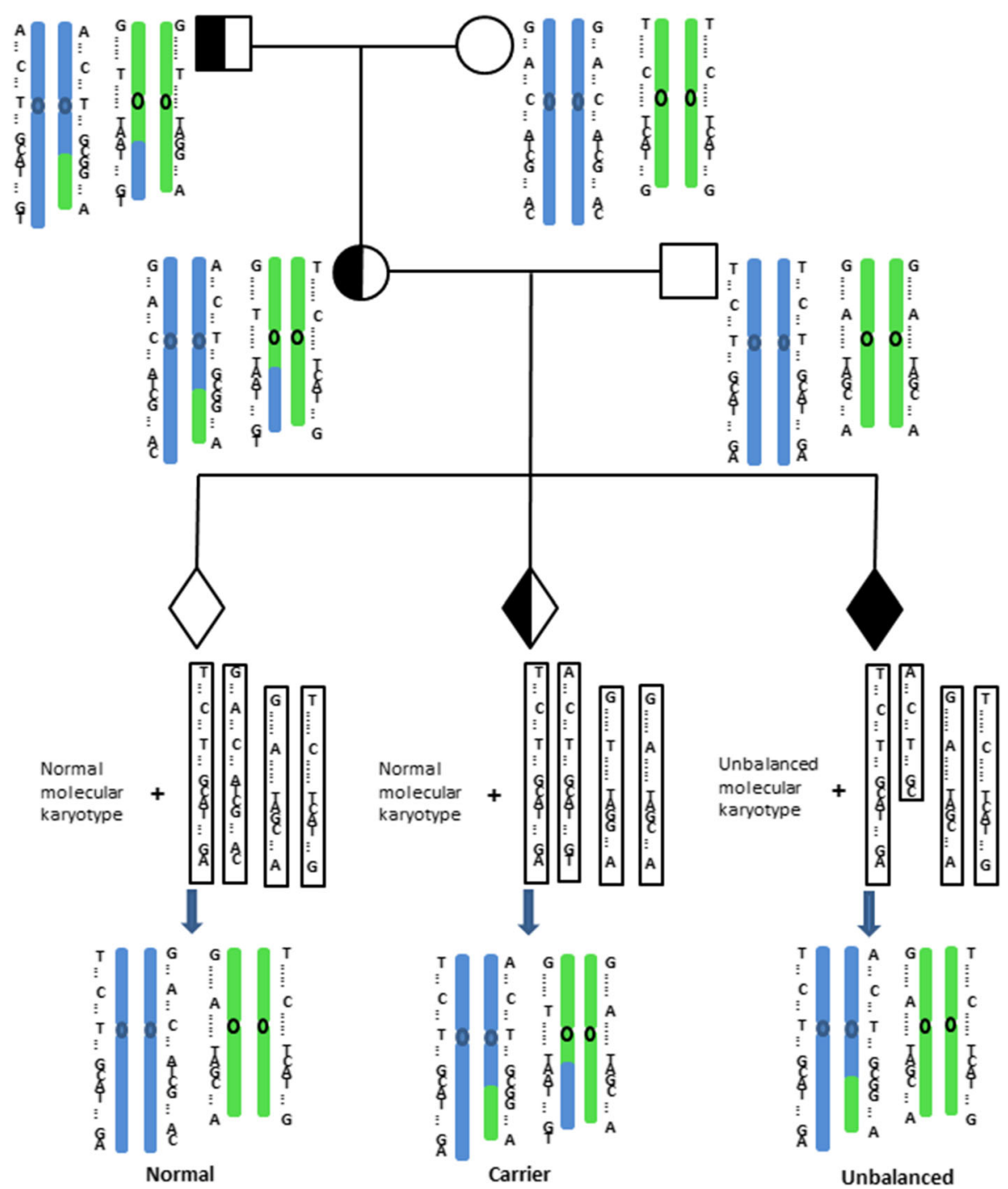

Fig. 1 The process of establishing haplotypes and distinguishing between balanced and structurally normal chromosomes embryos through PGH analysis. Informative SNPs should be heterozygous in the carrier, and homozygous in the carrier's partner and carrier's family member. These SNPS were used to establish the haplotypes of the breakpoint regions, the whole chromosomes involved in the translocation and the corresponding normal homologous chromosomes in the couple, reference and embryos

the predictive results of PGH and cytogenetic results of amniotic fluid cells were totally consistent. The sensitivity and specificity were $100 \%$, respectively.

\section{Discussion}

Despite having a successful PGD cycle and delivery in translocation families, many of these couples will be passing on the translocation to their children who may also be subjected to infertility, recurrent pregnancy loss or even have to seek assisted reproductive technologies to finally conceive. In our center, many couples express a strong desire to pursue more careful screening and transfer structurally normal embryos, especially for carriers with an abnormal phenotype. It is known that about $6 \%$ of translocation carriers present with a series of symptoms such as autism, mental retardation, or congenital abnormalities [22, 23].

Many researchers have attempted to overcome the difficulty in determining which embryos are non-carriers of a familial translocation. Initially, FISH with chromosome specific probes spanning the translocation breakpoints were used to differentiate between normal or balanced embryos $[15,16]$. Although relatively feasible, the techniques used were extremely complicated and not suitable for routine clinical diagnosis. Treff and colleagues enrolled reciprocal translocation families and predicted the balanced or normal status of each embryo based 


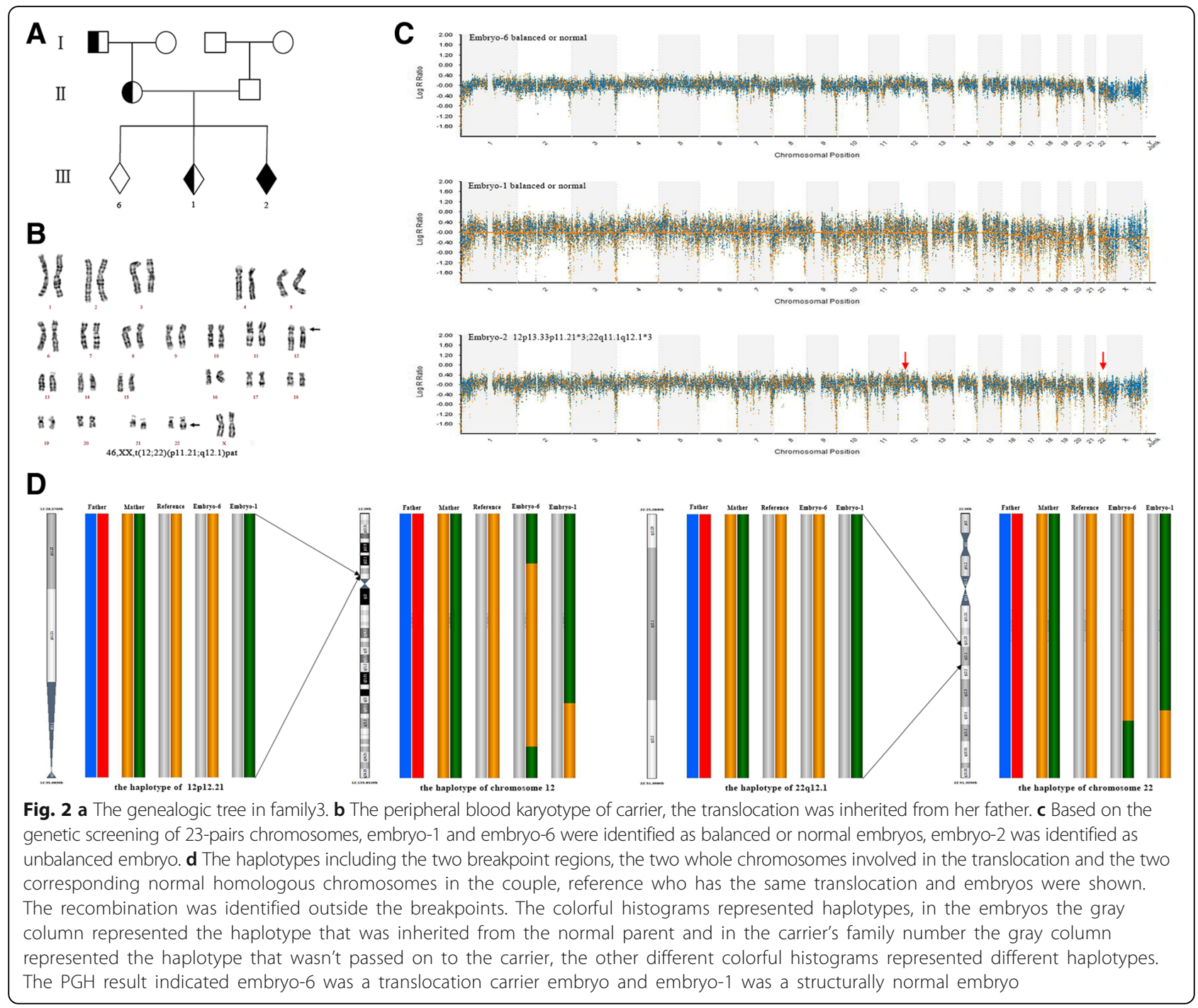

upon SNP genotypes within five $\mathrm{Mb}$ of the breakpoints using the Affymetrix NspI Gene Chip [17], in these cases the unbalanced embryos were the only reference used. In addition, in the latest research of $\mathrm{Hu}$ et al. [18], the authors developed the "MicroSeq-PGD" method which combined chromosome microdissection technique and NGS, to distinguish between balanced and structurally normal embryos in reciprocal translocation carriers by junction spanning PCR sequencing analysis and/or linkage analysis. This method could identify the translocation breakpoints and find the disrupted genes precisely. However, these current methods are time-consuming, complicated, and not available in most reproductive centers. More importantly, Robertsonian translocation carriers were not included, as the authors couldn't find the breakpoints which are located in the centromeric regions. Moreover, homologous recombination couldn't be identified using these methods.
Compared with these studies, several obvious advantages could be concluded in our research. First, the prediction for the status of chromosomes in embryos and the genetic screening for 23-pairs of chromosomes could be performed simultaneously using our method. Second, besides the haplotypes of the breakpoint regions, the haplotypes of the two whole chromosomes involved in the translocation and the two corresponding normal homologous chromosomes could be established in the carrier simultaneously, which could show the presence of homologous recombination in the breakpoint regions. Therefore, the prediction of PGH should combine the haplotypes of breakpoint regions and the whole chromosomes. Coincidentally, in this research, we didn't identify recombination in the breakpoint regions in all the embryos. Third, the haplotype of any region or chromosome genome wide could be established, so our method was universal for any kind of translocation. Fourth, the methodology was relatively 
Table 3 Summary of informative SNPs used to establish the whole haplotypes of the successfully transferred blastocysts

\begin{tabular}{|c|c|c|c|c|c|c|c|c|}
\hline Family $^{a}$ & $\begin{array}{l}\text { Number of } \\
\text { blastocysts }\end{array}$ & Chromosome & $\begin{array}{l}\text { The total number of } \\
\text { informative SNPs }\end{array}$ & $\begin{array}{l}\text { The average number of } \\
\text { informative SNPs } / \mathrm{Mb}\end{array}$ & $\begin{array}{l}\text { The number of } \\
\text { recombination SNPs }\end{array}$ & $\begin{array}{l}\text { The location of } \\
\text { recombination }\end{array}$ & $\begin{array}{l}\text { The location of } \\
\text { breakpoint }^{b}\end{array}$ & $\begin{array}{l}\text { Weather } \\
\text { recombination } \\
\text { occurs in the } \\
\text { breakpoint? }\end{array}$ \\
\hline \multirow[t]{2}{*}{1} & Embryo-1 & 5 & 1520 & 8.4 & 91 & 5:1-7,228,178(p15.33p15.31) & $5 q 33.1$ & No \\
\hline & & 22 & 401 & 7.8 & 0 & NR & $22 q 12$ & No \\
\hline \multirow[t]{2}{*}{2} & Embryo-4 & 16 & 823 & 9.1 & 148 & 16:1-9,724,564(p13.3p13.2) & $16 q 23.1$ & No \\
\hline & & 18 & 637 & 8.2 & 197 & $\begin{array}{l}\text { 18:7,105,507-31,960,623 } \\
\text { (p11.23q12.1) }\end{array}$ & $18 q 21.31$ & No \\
\hline \multirow[t]{3}{*}{3} & Embryo-6 & 12 & 1121 & 8.4 & 218 & $12: 1-25,329,895(p 13.33 p 12.1)$ & $12 \mathrm{p} 11.21$ & No \\
\hline & & & & & 163 & $\begin{array}{l}\text { 12:117,913,186-133,851,895 } \\
\text { (q24.22q24.33) }\end{array}$ & & No \\
\hline & & 22 & 419 & 8.2 & 149 & $\begin{array}{l}\text { 22:40,212,715-51,304,566 } \\
(q 13.1 q 13.33)\end{array}$ & $22 q 12.1$ & No \\
\hline \multirow[t]{2}{*}{4} & Embryo-1 & 11 & 1328 & 9.8 & 309 & $\begin{array}{l}\text { 11:94,616,073-127,991,048 } \\
\text { (q21 q24.3) }\end{array}$ & $11 p 11.2$ & No \\
\hline & & 16 & 648 & 7.2 & 209 & 16:1-16,599,806(p13.3p13.11) & $16 \mathrm{p} 12.3$ & No \\
\hline \multirow[t]{3}{*}{5} & Embryo-1 & 1 & 1802 & 7.2 & 763 & $\begin{array}{l}\text { 1:15,110,819-116,270,101 } \\
\text { (p36.21 p13.2) }\end{array}$ & $1 \mathrm{q} 21.2$ & No \\
\hline & & & & & 117 & $\begin{array}{l}1: 235,510,141-249,250,261 \\
(q 42.3 q 44)\end{array}$ & & No \\
\hline & & 19 & 482 & 8.2 & 115 & $\begin{array}{l}19: 51,465,236-59,128,983 \\
(q 13.41 \mathrm{q} 13.43)\end{array}$ & 19p13.11 & No \\
\hline \multirow[t]{2}{*}{6} & Embryo-9 & 14 & 672 & 6.3 & 0 & NR & Centromere & No \\
\hline & & 21 & 350 & 7.3 & 0 & NR & Centromere & No \\
\hline \multirow[t]{2}{*}{7} & Embryo-6 & 14 & 776 & 7.2 & 558 & $\begin{array}{l}14: 44,534,715-107,349,540 \\
(q 21.2 q 32.33)\end{array}$ & Centromere & No \\
\hline & & 21 & 363 & 7.5 & 0 & $N R$ & Centromere & No \\
\hline \multirow[t]{2}{*}{8} & Embryo-2 & 14 & 787 & 7.3 & 192 & $\begin{array}{l}14: 65,512,354-89,493,151 \\
(q 23.3 q 31.3)\end{array}$ & Centromere & No \\
\hline & & 15 & 785 & 7.7 & 0 & $N R$ & Centromere & No \\
\hline \multirow[t]{10}{*}{9} & Embryo-4c & 6 & 1565 & 9.2 & 197 & $\begin{array}{l}\text { 6:37,549,903-56,107,530 } \\
(\mathrm{p} 21.2 \mathrm{p} 12.1)\end{array}$ & $6 q 27$ & No \\
\hline & & & & & 58 & $\begin{array}{l}\text { 6:60,539,141-65,772,043 } \\
(q 11.1 q 12)\end{array}$ & & No \\
\hline & & & & & 19 & $\begin{array}{l}6: 85,870,071-88,787,897 \\
(q 14.3 q 15)\end{array}$ & & No \\
\hline & & & & & 40 & $\begin{array}{l}\text { 6:168,092,007-171,115,067 } \\
(q 27)\end{array}$ & & No \\
\hline & & 9 & 936 & 6.7 & 9 & $\begin{array}{l}9: 2,331,347-2,792,531 \\
(p 24.3 p 24.2)\end{array}$ & & No \\
\hline & & & & & 424 & $\begin{array}{l}\text { 9:5,098,509-77,784,684 } \\
\text { (p24.1q21.13) }\end{array}$ & $9 q 22$ & No \\
\hline & Embryo-4 ${ }^{\mathrm{d}}$ & 6 & 751 & 4.4 & 13 & $\begin{array}{l}\text { 6:11,558,464-14,305,025 } \\
\text { (p24.2p23) }\end{array}$ & $6 q 27$ & No \\
\hline & & & & & 197 & $\begin{array}{l}\text { 6:37,549,903-56,107,530 } \\
(\mathrm{p} 21.2 \mathrm{p} 12.1)\end{array}$ & & No \\
\hline & & & & & 57 & $\begin{array}{l}\text { 6:60,539,141-65,772,043 } \\
\text { (p11.1q12) }\end{array}$ & & No \\
\hline & & 9 & 367 & 2.6 & 179 & $\begin{array}{l}\text { 9:12,809,535-76,736,238 } \\
\text { (p23q21.13) }\end{array}$ & $9 q 22$ & $N A^{e}$ \\
\hline
\end{tabular}

$\mathrm{NR}=$ no recombination; $\mathrm{NA}=$ not available

an family10-11, as the unbalanced embryos were the only reference, the whole chromosome haplotypes couldn't be established

${ }^{\mathrm{b}}$ The breakpoints were identified by microarray results, except chromosome 22 in family 1

'The carrier's brother was used as a reference

${ }^{\mathrm{d}}$ The carrier's sister was used as a reference

'The haplotype couldn't be established in this breakpoint region, for no informative SNPs existed 
simple, including the experiment and date analysis, which was suitable for routine clinical work. The total process could be finished within two days. Fifth, chromosome translocation including reciprocal translocation and Robertsonian translocation, both these two types were able to be analyzed by our method.

Although the predictive results of unbalanced embryos were consistent with the family number in our study, we thought that not all the unbalanced embryos could be used as a reference (such as the unbalanced embryo \#5 and embryo \#13 in family 1). Here three copies in 5q33.1q35.3 were identified by molecular karyotyping and three haplotypes might potentially exist. It was therefore difficult to determine which of the carrier's haplotypes are linked to the derivative or to the normal chromosomes. Possibly, only the embryos with deletion of the translocation related fragments could be used. We analyzed semen from 9 male translation carriers with FISH and we found this kind of unbalanced sperm that contained only duplications of translocation related fragments accounted for $0.2 \% \sim 5.0 \%$ of the semen, while an abnormality was not identified in other 18 non- translation carriers semen, possibly the actual situation of meiosis in translocation carriers was much more complex than theoretically [24]. Besides, the unbalanced embryos couldn't help establish the haplotypes of whole chromosome, and therefore couldn't indicate whether there was recombination around the breakpoints. To some extent, the haplotype of the whole chromosome was even more important than that of breakpoint region in our opinion, for the former included the latter.

Meanwhile, we found, when the carrier's sister was use as reference in family-9, near $84 \mathrm{Mb}$ in chromosome 6 $(72440951-156,427,957)$ and $55 \mathrm{Mb}$ in chromosome 9 (85788944- 140,962,305) were same with the carrier besides the derivative chromosomes, therefore the haplotypes of these regions couldn't be established for lacking of informative SNPs. This might explain the small number of informative SNPs. It's supposed only half of the carrier's brothers and sisters could be used as reference although with the same translocation, theoretically the probability was $50 \%$ to inherit the different homologous chromosome except the derivative chromosome. Moreover, the called SNPs in microarray from biopsied cell DNA should be less than those from peripheral blood DNA. Overall, for the inherited families, the family member especially carrier's parents should be the preferred reference; for the de novo families, the unbalanced embryo would be also the choice. The complementarity of the two references might be the best option in clinical, which would be available for more translocation families undergoing IVF treatment. In the method of $\mathrm{Hu}$ and colleagues, although the authors didn't need a reference which was really exciting, two limitations were potential. First, the complexed technology was difficult to be widely popularized in clinical laboratories. Factually, identifying the translocation breakpoints precisely by microdissection was not necessary to predict the chromosome status in embryos. Second, Robertsonian translocation carriers which accounted for $24.3 \%$ of all translocation carriers in our center could not use this method.

In addition, we found the average rate of homologous recombination in derivative chromosomes had no differences with the normal chromosomes according to our results [21], meaning that the existing of the quadrivalent structure didn't reduce the chances of recombination between the paired translocated chromosomes and the two corresponding normal chromosomes. In each Mb distance, $6.6 \pm 1.4$ SNPs could be used to establish haplotypes, the recombination less than $1 \mathrm{Mb}$ also could be identified in the method.

\section{Conclusions}

In summary, with the validation and successful clinical application in our study, we proved that PGH is an efficient method to distinguish between balanced and structurally normal chromosome embryos from reciprocal and Robertsonian translocation carriers. This study has great clinical signification for these patients. More balanced translocation families would benefit from stopping the passing on of the translocation to their next generation. However, the sensitivity and specificity should be further validated in a larger sample size. Furthermore, PGH should also be used to distinguish between normal and inversion embryos from chromosome inversion carriers.

\section{Additional files}

Additional file 1: Table S1. Detailed results of microarray platform of non-transferred blastocysts. (DOC 114 kb)

Additional file 2: Table S2. Summary of informative SNPs used to establish the whole haplotypes of the non-transferred blastocysts. (DOC 86 kb)

\begin{abstract}
Abbreviations
CNV: Copy number variation; FISH: Fluorescent in-situ hybridization; IVF: In vitro fertilization; MDA: Multiple displacement amplification; PGD: Preimplantation genetic diagnosis; PGH: Preimplantation genetic haplotyping; SNP: Single nucleotide polymorphism; WGA: Whole genomic amplification
\end{abstract}

\section{Acknowledgements}

We express deepest gratitude to all the families enrolled in our research. We also thank the staff of the Genetics Laboratory and IVF Laboratory for their invaluable efforts and contribution relating to the experiment. Finally, we thank Dr. Harvey Stern (Director, Reproductive Genetics \& IVF Institute in Fairfax, USA) for his help on the manuscript preparation. 


\section{Availability of data and materials}

The datasets generated and analyzed during the current study are not publicly available due to a concern to protect individual patient confidentiality but are available from the corresponding author on reasonable request.

\section{Authors' contributions}

$\mathrm{SZ}, \mathrm{YZ}, \mathrm{DR}$ and $\mathrm{XS}$ designed the research and wrote the manuscript; $\mathrm{SZ}$ and $\mathrm{CL}$ performed the bioinformatics analysis; SZ, JW, JZ and HS performed the microarray experiments; JZ performed cytogenetic experiments of amniotic fluid cell and blood. JF and YS performed the intra-cytoplasmic sperm injection and blastocyst biopsy experiments; $\mathrm{SZ}$ and $\mathrm{YZ}$ collected the cases. All authors have read and approved the final manuscript.

\section{Ethics approval and consent to participate}

Written informed consent was obtained from each family and this study was approved by the Ethics Committee for Human Subject research of the Obstetrics and Gynecology Hospital, Fudan University.

\section{Consent for publication}

We obtained the consent to publish their clinical data from the patients in this study.

\section{Competing interests}

The authors declare that they have no competing interests. Daru Lu, one of the authors, is the member of the Editorial Board of this journal.

\section{Publisher's Note}

Springer Nature remains neutral with regard to jurisdictional claims in published maps and institutional affiliations.

\section{Author details}

'Shanghai Ji Ai Genetics \& IVF Institute, Obstetrics and Gynecology Hospital, Fudan University, 588 Fangxie Rd, Shanghai 200011, China. ${ }^{2}$ Obstetrics and Gynecology Hospital, Fudan University, 588 Fangxie Rd, Shanghai 200011, China. ${ }^{3}$ Collaborative Innovation Center for Genetics and Development, School of Life Sciences, Fudan University, 2005 Songhu Rd, Shanghai 200438, China.

Received: 9 April 2017 Accepted: 2 October 2017

Published online: 17 October 2017

\section{References}

1. Stern C, Pertile M, Norris H, Hale L, Baker HW. Chromosome translocations in couples with in-vitro fertilization implantation failure. Hum Reprod. 1999:14:2097-101.

2. Alfarawati $S$, Fragouli $E$, Colls $P$, Wells $D$. First births after preimplantation genetic diagnosis of structural chromosome abnormalities using comparative genomic hybridization and microarray analysis. Hum Reprod. 2011:26:1560-74.

3. Lim CK, Cho JW, Song IO, Kang IS, Yoon YD, Jun JH. Estimation of chromosomal imbalances in preimplantation embryos from preimplantation genetic diagnosis cycles of reciprocal translocations with or without acrocentric chromosomes. Fertil Steril. 2008;90:2144-51.

4. Fiorentino F, Spizzichino L, Bono S, Biricik A, Kokkali G, Rienzi L, et al. PGD for reciprocal and Robertsonian translocations using array comparative genomic hybridization. Hum Reprod. 2011;26:1925-35.

5. Zhang YP, Zhu SJ, JL W, Sun XX. Quadrivalent asymmetry in reciprocal translocation carriers predicts meiotic segregation patterns in cleavage stage embryos. Reprod BioMed Online. 2014;29:490-8.

6. Beyazyurek C, Ekmekci CG, Sağlam Y, Cinar C, Kahraman S. Preimplantation genetic diagnosis (PGD) for extremes-successful birth after PGD for a consanguineous couple carrying an identical balanced reciprocal translocation. Fertil Steril. 2010;93:1-5.

7. Ko DS, Cho JW, Park SY, Kim JY, Koong MK, Song IO, et al. Clinical outcomes of preimplantation genetic diagnosis (PGD) and analysis of meiotic segregation modes in reciprocal translocation. Am J Med Genet A. 2010;152A:1428-33.

8. Fiorentino F, Kokkali G, Biricik A, Stavrou D, Ismailoglu B, De Palma R, et al. Polymerase chain reaction-based detection of chromosomal imbalances on embryos: the evolution of preimplantation genetic diagnosis for chromosomal translocations. Fertil Steril. 2010;94:2001-11.
9. Tan Y, Yin X, Zhang S, Jiang H, Tan K, Li J, et al. Clinical outcome of preimplantation genetic diagnosis and screening using next generation sequencing. GigaScience. 2014;3:1-9.

10. Yang Z, Lin J, Zhang J, Fong WI, Li P, Zhao R, et al. Randomized comparison of next-generation sequencing and array comparative genomic hybridization for preimplantation genetic screening: a pilot study. Bmc Medical Genomics. 2015;8:30

11. Huang CC, Chang LJ, Tsai YY, Hung CC, Fang MY, YN S, et al. A feasible strategy of preimplantation genetic diagnosis for carriers with chromosomal translocation: Using blastocyst biopsy and array comparative genomic hybridization. J Formos Med Assoc. 2013;112:537-44.

12. Tan YQ, Tan K, Zhang SP, Gong F, Cheng DH, Xiong B, et al. Single-nucleotide polymorphism microarray-based preimplantation genetic diagnosis is likely to improve the clinical outcome for translocation carriers. Hum Reprod. 2013:28:2581-92

13. Colls P, Escudero T, Fischer J, Cekleniak NA, Ben-Ozer S, Meyer B, et al. Validation of array comparative genome hybridization for diagnosis of translocations in preimplantation human embryos. Reprod BioMed Online. 2012;24:621-9.

14. Uum CMV, Stevens SJ, Dreesen JC, Drüsedau M, Smeets HJ, HollandersCrombach B, et al. SNP array-based copy number and genotype analyses for preimplantation genetic diagnosis of human unbalanced translocations. Eur J Hum Genets. 2012;20:938-44.

15. Weier HU, Munn S, Fung J. Patient-specific probes for preimplantation genetic diagnosis of structural and numerical aberrations in interphase cells. J Assist Reprod Genet. 1999:16:182-91.

16. Lersch RA, Fung J, Munné S, Pedersen RA, Weier HU. Case-Specific, Breakpoint- Spanning DNA Probes for Analysis of Single Interphase Cells. Genet Test. 2000;4:273-8.

17. Treff NR, Thompson K, Rafizadeh M, Chow M, Morrison L, Tao X, et al. SNP array-based analyses of unbalanced embryos as a reference to distinguish between balanced translocation carrier and normal blastocysts. J Assist Reprod Genet. 2016:33:1-5

18. Hu L, Cheng D, Gong F, Lu C, Tan Y, Luo K, et al. Reciprocal Translocation Carrier Diagnosis in Preimplantation Human Embryos. EBioMedicine. 2016;14:139-47

19. Hamerton JL, Canning N, Ray M, Smith S. A cytogenetic survey of 14,069 newborn infants : I. Incidence of chromosome abnormalities. Clin Genet. 1975:8:223-43.

20. Renwick PJ, Trussler J, Ostadsaffari E, Fassihi H, Black C, Braude P, et al. Proof of principle and first cases using preimplantation genetic haplotyping-a paradigm shift for embryo diagnosis. Reprod BioMed Online. 2006;13:110-9.

21. Handyside AH, Harton GL, Mariani B, Thornhill AR, Affara N, Shaw MA, et al. Karyomapping: a universal method for genome wide analysis of genetic disease based on mapping crossovers between parental haplotypes. J Med Genet. 2010:47:651-8.

22. Bugge M, Bruunpetersen G, Brøndumnielsen K, Friedrich U, Hansen J, Jensen $G$, et al. Disease associated balanced chromosome rearrangements: a resource for large scale genotype-phenotype delineation in man. J Med Genet. 2000;37:858-65.

23. Tonk VS, Wyandt HE, Huang X, Patel N, Morgan DL, Kukolich M, et al. Disease associated balanced chromosome rearrangements (DBCR): report of two new cases. Ann Genet. 2003:46:37-43.

24. Scriven PN, Handyside AH, Ogilvie CM. Chromosome translocations: segregation modes and strategies for preimplantation genetic diagnosis. Prenat Diagn. 1998;18(supplement):1437-49. 